\title{
THE EXCHANGE OF MASSLESS SPIN-TWO PARTICLES
}

\author{
J.J. VAN DER BIJ* and H. VAN DAM** \\ Instituut voor Theoretische Fysica, Rijksuniversiteit Utrecht, Princetonplein 5, P. O. Box 80.006, \\ 3508 TA Utrecht, The Netherlands \\ and \\ YEE JACK NG*** \\ Institute of Field Physics, Department of Physics and Astronomy University of North Carolina, \\ Chapel Hill, NC 27514, USA
}

Received 17 May 1982

The theory of gravity is considered from the little group viewpoint. This leads to a theory with a constraint, which is equivalent to general relativity with an arbitrary cosmological term. With this framework (i) the cosmological constant cannot be put into the Lagrangian but it appears as an integration constant. (ii) The gravitational Lagrangian automatically takes the form of a finite polynomial of the metric. (iii) The so-called conformal factor is fixed, which removes an apparent difficulty in carrying out path integrals.

\section{Introduction}

The quantum description of massless particles of spin 1 and higher leads in general to the introduction of gauge invariance. This is due to the fact that the subgroup of the Poincaré group which leaves the four momentum of a free massless particle invariant (the so called little group) is not compact. In this paper we discuss the quantum description of the graviton from the point of view of the little group.

In section 2, we show, starting from the description of massless spin two particles, as given by Wigner ${ }^{1}$ ), that one is led to consider gauge transformations of the form $h_{\mu \nu} \rightarrow h_{\mu \nu}+\partial_{\mu} \xi_{\nu}+\partial_{\nu} \xi_{\mu}$, with $\xi_{\mu}$ constrained to $\partial_{\mu} \xi_{\mu}=0$. The usual approach ${ }^{2}$ ) lacks this constraint.

On the basis of invariance for these constrained gauge transformations we derive in section 3 a Lagrangian for the free graviton. We contrast this Lagrangian with that of the linearized theory of gravity.

In section 4 we derive a propagator for the graviton from this Lagrangian.

* Present address: Physics Dept., University of Michigan, Ann Arbor, MI 48109, USA.

** On leave of absence from the University of North Carolina, Chapel Hill, NC.

****Alfred P. Sloan fellow. 
Using this propagator, we show that only massless spin two particles are exchanged by gauge-invariant sources, so that the theory is free of ghosts. In a surprising way, these sources can only differ from the energy-momentum tensor for matter by an arbitrary constant factor. The exchange can be written exactly as in the usual linearized theory of gravity and leads to confirmation of the local tests of that theory.

In section 5 we extend the gauge transformation of the linearized theory to the group of volume-preserving general coordinate transformations and we give a Lagrangian for the full theory. The solutions to these equations all satisfy the constraint $-\operatorname{det}\left(g_{\mu \nu}\right)=1$ and Einstein's equation for some value of the cosmological constant $\Lambda$ (different values of $\Lambda$, in general, for different solutions). In the framework presented this cosmological constant $\Lambda$ enters as an arbitrary constant of integration, rather than as a term in the action. This provides a somewhat different point of view which may be an advantage, for instance, in handling vacuum expectation values of the energy-momentum tensor, such as occur for Higgs fields ${ }^{3}$ ). We finish section 5 with an argument that any solution to Einstein's equations allows one to choose a coordinate system in which det $g_{\mu \nu}=-1$.

In section 6 we discuss several furtherproperties of the proposed framework. Among which:

a) There is no negative and arbitrarily large conformal factor contribution to the Euclidean Action.

b) The Lagrangian has the form of a finite polynomial in the metric, with a constraint, providing a link with $\sigma$-models.

c) From our point of view the disagreement over the measure in the space of all metrics, which exists in the literature, seems to be irrelevant.

2. The quantum description of a single massless particle of spin 2, gauge transformations: the little group viewpoint.

Within the framework of invariance for the Poincare group (inhomogeneous proper orthochronous Lorentz group) the description of a particle with definite mass and spin was completely determined by Wigner ${ }^{1}$ ). For a massless particle the appropriate unitary representation of the covering group of the "little group" of the fourvector

$$
k=(0,0, E, \mathrm{i} E), E>0 .
$$

This little group is the subgroup of the Lorentzgroup which leaves the fourvector (1) invariant, its generators are the rotation around the $z$-axis, 


$$
I_{12}=\left(\begin{array}{rrrr}
0 & 1 & 0 & 0 \\
-1 & 0 & 0 & 0 \\
0 & 0 & 0 & 0 \\
0 & 0 & 0 & 0
\end{array}\right)
$$

and the "translations"

$$
I_{13}-I_{14}=\left(\begin{array}{rrrr}
0 & 0 & 1 & 1 \\
0 & 0 & 0 & 0 \\
-1 & 0 & 0 & 0 \\
-1 & 0 & 0 & 0
\end{array}\right), \quad I_{23}-I_{24}=\left(\begin{array}{rrrr}
0 & 0 & 0 & 0 \\
0 & 0 & 1 & 1 \\
0 & -1 & 0 & 0 \\
0 & -1 & 0 & 0
\end{array}\right)
$$

These three generators generate a group which is isomorphic to the group of the two-dimensional Euclidean plane. That is the reason for calling two of the generators "translations". Unless these translations are represented trivially, the unitary representation of the covering group is infinite dimensional. Such an infinite-dimensional representation would mean that for given fourmomentum $k$ there are an infinite number of orthogonal states, i.e. an infinite spin and an infinite specific heat. In this way one is led to the demand that the translation like generators of the little group leave the states of fixed $k$ invariant. This demand is the source of the "gauge invariance", ), we illustrate it for spin 1 and for spin 2.

Consider first a massless spin-1 particle (say, the photon) with momentum given by (1) and polarization vectors $e^{(1)}=(1,0,0,0)$ and $e^{(2)}=(0,1,0,0)$. Under $I_{12} e^{(1)}$ and $e^{(2)}$ rotate into each other; but $e^{(1)}$ changes under $I_{13}-I_{14}$ and $e^{(2)}$ changes under $I_{23}-I_{24}$, they "gauge" according to

$$
e^{(1)}+\lambda(0,0, E, \mathrm{i} E), \quad e^{(2)} \rightarrow e^{(2)}+\lambda^{\prime}(0,0, E, \mathrm{i} E) .
$$

Hence, the two polarization states of a photon cannot be described by two unit vectors. They can, however, be described by the equivalence classes of two unit vectors:

$$
\left\{e^{(1)}+\lambda(0,0, E, i E)\right\}, \quad\left\{e^{(2)}+\lambda^{\prime}(0,0, E, i E)\right\} .
$$

For a general value of $k_{\mu}$ the two polarization states of the photon are described by two equivalence classes of two unit vectors:

$$
\left\{e_{\mu}^{(i)}(k)+\lambda(k) k_{\mu} \mid \text { for all } \lambda(k) \text { such that } k^{2} \lambda(k)=0\right\}
$$

where $i=1,2$, and where

$$
k_{\mu} e_{\mu}^{(1)}=0=k_{\mu} e_{\mu}^{(2)}, \quad e_{\mu}^{(1)} e_{\mu}^{(2)}=0 .
$$

The mass shell constraint $\square \lambda=0$, contained in (5a), is too restrictive for interaction and is dropped, leading to the usual gauge invariance under the 
transformation

$$
A_{\mu} \rightarrow A_{\mu}+\partial_{\mu} \lambda
$$

where $\lambda(x)$ is an arbitrary function.

Consider next a massless spin two particle. Naively one might want to describe the two polarization states of a massless spin-two particle, with fourmomentum given by (1), by the two symmetric tensors

$$
\sqrt{2} e_{\mu \nu}^{(1)}=\left(\begin{array}{rrrr}
1 & 0 & 0 & 0 \\
0 & -1 & 0 & 0 \\
0 & 0 & 0 & 0 \\
0 & 0 & 0 & 0
\end{array}\right), \quad \sqrt{2} e_{\mu \nu}^{(2)}=\left(\begin{array}{cccc}
0 & 1 & 0 & 0 \\
1 & 0 & 0 & 0 \\
0 & 0 & 0 & 0 \\
0 & 0 & 0 & 0
\end{array}\right)
$$

For the rotations $I_{12}$ these two states have the correct spin-two behavior. However, they are not invariant for $I_{13}-I_{14}$, or for $I_{23}-I_{24}$, in fact by these the $e_{\mu \nu}^{(i)}$ are changed into

$$
\left(\begin{array}{cccc}
1 & 0 & \lambda & \mathrm{i} \lambda \\
0 & -1 & \lambda^{\prime} & -\mathrm{i} \lambda^{\prime} \\
\lambda & -\lambda^{\prime} & \lambda^{2}-\lambda^{\prime 2} & \mathrm{i}\left(\lambda^{2}-\lambda^{\prime 2}\right) \\
\mathrm{i} \lambda & -\mathrm{i} \lambda^{\prime} & \mathbf{i}\left(\lambda^{2}-\lambda^{\prime 2}\right) & -\left(\lambda^{2}-\lambda^{\prime 2}\right)
\end{array}\right) \text { and }\left(\begin{array}{cccc}
0 & 1 & \lambda^{\prime} & \mathrm{i} \lambda^{\prime} \\
1 & 0 & \lambda & \mathrm{i} \lambda \\
\lambda^{\prime} & \lambda & 2 \lambda \lambda^{\prime} & 2 \mathrm{i} \lambda \lambda^{\prime} \\
\mathbf{i} \lambda^{\prime} & \mathbf{i} \lambda & 2 \mathrm{i} \lambda \lambda^{\prime} & -2 \lambda \lambda^{\prime}
\end{array}\right)
$$

Note that the tensors (8) are still traceless. The states of the massless spin-two particle are described not by (7), but by the two equivalence classes

$$
\left\{e_{\mu \nu}^{(i)}(k)+V_{\mu} k_{\nu}+V_{\nu} k_{\mu} \mid \text { for all } V \text { so that } k_{\mu} V_{\mu}=0 \text { and } k^{2} V_{\mu}=0\right\},
$$

where $i=1,2$,

$$
k_{\mu} e_{\mu \nu}^{(i)}(k)=0, \quad e_{\mu \nu}^{(1)}(k) e_{\mu \nu}^{(2)}(k)=0, \quad e_{\mu \nu}^{(i)}(k)=e_{\nu \mu}^{(i)}(k),
$$

and where the $e_{\mu \nu}^{(i)}$ are traceless:

$$
e_{\mu \mu}^{(i)}(k)=0 .
$$

This tracelessness is a property of all the members of the equivalence class of (9a), the trace is preserved by the little group transformations (2).

Hence we describe the states of the particle by the traceless symmetric tensor field $h_{\mu \nu}(k)$, which satisfies $h_{\mu \mu}(k)=0=k_{\mu} h_{\mu \nu}$, and which has a gauge invariance

$$
h_{\mu \nu}(k) \rightarrow h_{\mu \nu}(k)+k_{\mu} \xi_{\nu}+k_{\nu} \xi_{\mu},
$$

with two constraints, $k^{2} \xi_{\mu}(k)=0$, and

$$
k_{\mu} \xi_{\mu}=0 \text {. }
$$

The first of these constraints is analogous to the single constraint which 
appeared for the spin-one case. It has to be dropped as, again, it is too restrictive for interaction. At this point one usually drops this constraint as well as the constraint (11) and is then lead, fairly straightforwardly, to general relativity ${ }^{5}$ ). We see no immediate reason to drop (11), which means we have three free functions instead of the usual four. Unlike the mass shell constraint, (11) has a geometric meaning (it suppresses a compression mode).

\section{Lagrangian in weak field approximation (linearized theory)}

This linearized theory is just a first approximation, interaction changes the commutative gauge group (10), (11), into the noncommutative "group" of (volume-preserving) general coordinate transformations with determinant 1. The full theory will be discussed in section 5 , here we limit ourselves to the linearized first order theory.

First, let us recall the action for the linearized theory of gravity, it is given by [2]

$$
\begin{aligned}
\mathscr{L}_{\mathrm{E}}= & -\frac{1}{2}\left(\partial_{\lambda} h_{\mu \nu} \partial_{\lambda} h_{\mu \nu}-\partial_{\lambda} h_{\alpha \alpha} \partial_{\lambda} h_{\beta \beta}\right)-\partial_{\mu} h_{\mu \nu} \partial_{\nu} h_{\alpha \alpha} \\
& +\frac{1}{2}\left(\partial_{\mu} h_{\mu \nu} \partial_{\lambda} h_{\lambda \nu}+\partial_{\lambda} h_{\mu \nu} \partial_{\mu} h_{\lambda \nu}\right)+\kappa T_{\mu \nu} h_{\mu \nu}
\end{aligned}
$$

This Lagrangian is invariant for $h_{\mu \nu} \rightarrow \xi_{\mu, \nu}+\xi_{\nu, \mu}+h_{\mu \nu}$, with $\xi_{\mu}$ free and not restricted by (11), provided that

$$
\partial_{\mu} T_{\mu \nu}=0 .
$$

To be more specific, let us consider the interaction with a scalar field $\phi$, then one must add to $\mathscr{L}_{\mathrm{E}}$ the Lagrangian for that field:

$$
\mathscr{L}_{\phi}=-\frac{1}{2} \partial_{\mu} \phi \partial_{\mu} \phi-V(\phi),
$$

where for the moment $V(\phi)=\frac{1}{2} m^{2} \phi^{2}$, and where $T_{\mu \nu}$ in (12) is given by

$$
T_{\mu \nu}=\frac{1}{2} \partial_{\mu} \phi \partial_{\nu} \phi-\frac{1}{2} \delta_{\mu \nu}\left(\frac{1}{2} \partial_{\alpha} \phi \partial_{\alpha} \phi+V(\phi)\right)
$$

That $T_{\mu v}$ satisfies (13) follows from the equation of motion for $\phi$ up to first order in $\kappa$ :

$$
\square \phi-V^{\prime}(\phi)=0+\mathcal{O}(\kappa) .
$$

The alternative theory which we wish to consider may be obtained by varying $h_{\mu \nu}$ in (12) not freely, but restricted to $h_{\alpha \alpha}=0$, which is done most easily by adding a Lagrange multiplier. One may also start instead of with (12) with

$$
\mathscr{L}=-\frac{1}{4} \partial_{\lambda} h_{\mu \nu} \partial_{\lambda} h_{\mu \nu}+\frac{1}{2}\left(\partial_{\lambda} h_{\lambda \mu}\right)^{2}+\kappa h_{\mu \nu} S_{\mu \nu}+\left[L h_{\alpha \alpha}\right] .
$$


Here the term in square brackets is a Lagrange multiplier. The action obtained from the first two terms on the right-hand side of (17) is invariant for (10) with constraint (11). Gauge invariance for the contribution to the action of the third term on the right-hand side implies that for some scalar $A(x)$

$$
\partial_{\mu} S_{\mu \nu}=\partial_{\nu} A \text {. }
$$

For the interaction with a scalar field one adds the Lagrangian $\mathscr{L}_{\phi}$ of (14) and substitutes e.g.

$$
S_{\mu \nu}=\frac{1}{2} \partial_{\mu} \phi \partial_{\nu} \phi .
$$

Then by varying $\phi, h_{\mu \nu}$ and $L$ independently, the equations of motion of $\phi$ are again (16), and those for $h$ are

$$
-\square h_{\mu \nu}+\partial_{\mu} \partial_{\lambda} h_{\lambda \nu}+\partial_{\nu} \partial_{\lambda} h_{\lambda \mu}-\frac{1}{2} \delta_{\mu \nu} \partial_{\alpha} \partial_{\beta} h_{\alpha \beta}=\kappa\left(S_{\mu \nu}-\frac{1}{4} \delta_{\mu \nu} S_{\alpha \alpha}\right),
$$

where $S_{\mu \nu}$ is given by (19). With (16) one calculates

$$
\partial_{\mu} S_{\mu \nu}=\frac{1}{2} V^{\prime}(\phi) \partial_{\nu} \phi+\partial_{\nu}\left(\partial_{\mu} \phi \partial_{\mu} \phi\right)+\mathcal{O}(\kappa) ;
$$

this ensures invariance for (10), (11), up to first order in $\kappa$.

Note that whereas the equations of motion of linearized gravity when coupled to $\phi$ involve $V(\phi)$ itself through (15) in (12), the eqs. (19), (20) and (16) involve only $V^{\prime}(\phi)$.

\section{Propagator, particle content}

For the linearized Einstein Lagrangian (12) the calculation of the propagator is straightforward ${ }^{2}$ ). The exchange of a single graviton between two conserved sources $T_{\mu \nu}$, and $t_{\alpha \beta}$ is given by ${ }^{2,6}$ )

$$
\mathscr{L}_{\text {int }}=\kappa^{2} \frac{\left(g_{\mu \alpha} g_{\nu \beta}+g_{\mu \beta} g_{\nu \alpha}-g_{\mu \nu} g_{\alpha \beta}\right)}{k^{2}-i \epsilon} T_{\mu \nu}(k) t_{\alpha \beta}(-k) .
$$

The residue at the pole $k^{2}=0$ gives one that the particles exchanged are the two polarization states of a massless spin two-particle. Also (22) explains all the local tests of general relativity: bending of light by the sun, delay of radar echoes, perihelion procession ${ }^{7}$ ), and also the gyroscope precession ${ }^{8}$ ).

Let us now investigate the restricted theory of Lagrangian (17). The gauge invariance of (17) is characterized by three functions instead of by the four of (12). However as the trace of $h$ is zero for (16) both theories (12), (17) allow a "zerodiagonal gauge":

$$
h_{\alpha \alpha}=0, \quad \alpha=1,2,3,4,
$$


where one does not sum over $\alpha$. To calculate the propagator corresponding to (17) in this zero diagonal gauge we add to (17) a gauge breaking term: $b \Sigma_{i=1}^{3} h_{i i} h_{i i}, b \rightarrow \infty$. In the limit $b \rightarrow \infty$ calculating the propagator consists of taking the inverse of a $6 \times 6$ symmetric matrix $\left.{ }^{9}\right), V_{\alpha \beta, \mu \nu}$, where $\alpha, \beta, \mu, \nu=1,2$, $3,4, \alpha \neq \beta, \mu \neq \nu$. The elements of this matrix follow by permutation from

$$
2 V_{12,12}=k^{2}-k_{1}^{2}-k_{2}^{2} ; \quad 2 V_{12,13}=-k_{2} k_{3} ; \quad V_{1234}=0 .
$$

For instance, $V_{12,23}=V_{21,23}=-k_{1} k_{3}$, etc. Thus for $b \rightarrow \infty$, the propagator $P_{\mu \nu, \alpha \beta}$ may be given by a $10 \times 10$ matrix of which all elements $\mu=\nu$, and/or $\alpha=\beta$ are zero. The elements of the remaining $6 \times 6$ symmetric matrix may be obtained by permutation from

$$
\begin{aligned}
& 2 P_{12,12}=\frac{1}{k^{2}} \frac{\left(k_{1}^{2}+k_{2}^{2}\right)^{2}}{k_{1}^{2} k_{2}^{2}} . \\
& 2 P_{12,13}=-\frac{1}{k^{2}} \frac{1}{k_{1}^{2} k_{2} k_{3}}\left[\left(k_{1}^{2}+k_{2}^{2}\right)\left(k_{1}^{2}+k_{3}^{2}\right)-2 k_{2}^{2} k_{3}^{2}\right], \\
& 2 P_{12,34}=-\frac{1}{k^{2}} \frac{\left(k_{1}^{2}+k_{2}^{2}\right)\left(k_{3}^{2}+k_{4}^{2}\right)}{k_{1} k_{2} k_{3} k_{4}} .
\end{aligned}
$$

For instance, $P_{12,24}=P_{21,23}$ is obtained from $P_{12,13}$ by $1 \leftrightarrow 2$, etc.

To find out what is being exchanged between two sources $S_{\mu \nu}$, and $s_{\alpha \beta}$ by (25), we calculate the residue at the pole $k^{2}=0$ of $S_{\mu \nu} P_{\mu \nu, \alpha \beta} s_{\alpha \beta}$, where because of Lorentz invariance we may take $k$ to be given by (1), and where

$$
k_{\mu} S_{\mu \nu}=k_{\nu} A(k), \quad k_{\alpha} s_{\alpha \beta}=k_{\beta} a(k),
$$

with $k$ given by (1), (26) reduces to

$$
\begin{array}{ll}
S_{12}+\mathrm{i} S_{14}=0, & S_{23}+\mathrm{i} S_{24}=0, \\
S_{33}+\mathrm{i} S_{34}=A, & S_{43}+\mathrm{i} S_{44}=\mathrm{i} A,
\end{array}
$$

and similarly for $s$ and $a$. With (27) and (25) we find for the residue $S_{11} s_{11}+S_{22} s_{22}+2 S_{12} s_{12}$, which equals $S_{\mu \nu}(k) s_{\alpha \beta}(-k)\left(\Sigma_{i=1}^{2} e_{\mu \nu}^{(i)}(k) e_{\alpha \beta}^{(i)}(k)\right)$, with $e_{\mu \nu}^{(i)}(k)$ given by (7). Hence what is being exchanged between $S$ and $s$ are the two polarization states of a massless spin two-particle.

For arbitrary values of $k^{2}$ the propagator with sources $S_{\mu \nu}(k), s_{\alpha \beta}(-k)$ is

$$
\begin{aligned}
& S_{\mu \nu}(k) P_{\mu \nu, \alpha \beta}(k) s_{\alpha \beta}(-k) \\
& \quad=\frac{2 S_{\mu \nu} s_{\mu \nu}-S_{\mu \mu} s_{\alpha \alpha}+2\left(A s_{\alpha \alpha}+a S_{\mu \mu}\right)-8 A a}{2\left(k^{2}-i \epsilon\right)} \\
& =\frac{2\left(S_{\mu \nu}-A \delta_{\mu \nu}\right)\left(s_{\mu \nu}-a \delta_{\mu \nu}\right)-\left(S_{\mu \mu}-A \delta_{\mu \mu}\right)\left(s_{\alpha \alpha}+a \delta_{\alpha \alpha}\right)}{2\left(k^{2}-i \epsilon\right)} .
\end{aligned}
$$

Note that with A from (18) 


$$
\tilde{T}_{\mu \nu}=S_{\mu \nu}-A \eta_{\mu \nu} \text { is conserved: } \partial_{\nu} \tilde{T}_{\mu \nu}=0 .
$$

In fact $\tilde{T}_{\mu \nu}=T_{\mu \nu}+c \delta_{\mu \nu}$, where $c$ is an arbitrary integration constant from solving (18) for $A$.

Eq. (28) shows that instead of coupling to the rather arbitrary $S_{\mu \nu}$ one should couple to $\tilde{T}_{\mu \nu}$ from the beginning, where $\tilde{T}_{\mu \nu}$ differs from the energy momentum tensor by a constant factor $c \delta_{\mu \nu}$.

\section{Non-weak field case (full theory)}

The linearized theory of gravity is just a first approximation. Starting from (12) and by introducing $g_{\mu \nu}=\delta_{\mu \nu}+\kappa h_{\mu \nu}$, one can bootstrap oneself to the full Einstein theory ${ }^{5}$ ). One can proceed with the Lagrangian (17) in a similar way. Along the way the commutative gauge transformations are changed into the infinitesimal (volume-preserving) general coordinate transformations of determinant 1 .

The simplest way to obtain the full theory is, however, to take the action of Einstein's theory and to limit the variations of $g_{\mu \nu}$ to-det $\left(g_{\mu \nu}\right) \equiv g=1$. (At this point we change notation from the metric $\delta_{\mu \nu}$ to $g_{\mu \nu}$ used in the context of general relativity).

Let us next compare explicitly, first, Einstein's theory of gravity, interacting with a scalar field and, second, the formulation proposed here interacting with a scalar field. A comparison similar to that made in section four for the linearized theories.

First, for Einstein's equations one has the action

$$
S=\int \mathrm{d} x \sqrt{g}\left[R-\frac{1}{2} g^{\mu \nu} \partial_{\mu} \phi \partial_{\nu} \phi-V(\phi)\right],
$$

where $V(\phi)$ can be $-\frac{1}{2} m^{2} \phi^{2}$, or some more complicated function of $\phi$. The equations of motion which follow from (30) are

$$
\begin{aligned}
& D^{\mu} D_{\mu} \phi-V^{\prime}(\phi)=0, \\
& R^{\mu \nu}-\frac{1}{2} g^{\mu \nu} R-T^{\mu \nu}=0 .
\end{aligned}
$$

where

$$
T^{\mu \nu}=\frac{1}{2} \partial^{\mu} \phi \partial^{\nu} \phi-\frac{1}{4} g^{\mu \nu} \partial^{\alpha} \phi \partial_{\alpha} \phi-\frac{1}{2} g^{\mu \nu} V(\phi) .
$$

The identity $D_{\mu}\left(R^{\mu \nu}-\frac{1}{2} g^{\mu \nu} R\right)=0$ gives the conservation law $D_{\mu} T^{\mu \nu}=0$ which is also a consequence of the first equation in (31).

Second, for the equations proposed here, we propose to take as an action

$$
S=\int \mathrm{d} x \sqrt{g}\left[R-\frac{1}{2} g^{\mu \nu} \partial_{\mu} \phi \partial_{\nu} \phi-V(\phi)+L(\sqrt{g}-1)\right],
$$


where $L(x)$ is a Lagrange multiplier, which is to be varied independently of $g_{\mu \nu}$ and $\phi$. Varying $L(x), \phi(x)$ and $g_{\mu \nu}(x)$ independently in (33) gives the equations

$$
\begin{aligned}
& g \equiv-\operatorname{det}\left(g_{\mu \nu}\right)=1, \\
& D^{\mu} D_{\mu} \phi-V^{\prime}(\phi)=0, \\
& R^{\mu \nu}-\frac{1}{2} g^{\mu \nu} R-T^{\mu \nu}=\frac{1}{2} L g^{\mu \nu},
\end{aligned}
$$

where $T^{\mu \nu}$ is given by (32). One may now solve for $L(x)$ by taking the trace of (36), which gives

$$
-R-T=2 L \text {. }
$$

Substituting back into (36) gives us the field equations for the action (33) with

$$
S^{\mu \nu}=\partial^{\mu} \phi \partial^{\nu} \phi
$$

as

$$
\begin{aligned}
& D^{\mu} D_{\mu} \phi-V^{\prime}(\phi)=0 \quad \text { or } \quad \partial^{\mu} \partial_{\mu} \phi-V^{\prime}(\phi)=0 \quad\left(\Gamma_{\mu \alpha}^{\mu}=0\right), \\
& -\operatorname{det}\left(g_{\mu \nu}\right)=1, \\
& R^{\mu \nu}-\frac{1}{4} g^{\mu \nu} R-S^{\mu \nu}+\frac{1}{4} g^{\mu \nu} S=0 .
\end{aligned}
$$

It is of use for our later considerations (polynomial form of Lagrangian) to replace the action (33) with an equivalent action which gives the same equations of motion (38), (37), it is

$$
S=\int \mathrm{d} x\left[R-\frac{1}{2} g^{\mu \nu} \partial_{\mu} \phi \partial_{\nu} \phi-V(\phi)+L(g-1)\right] .
$$

An important difference between (31) and (38) is that (via (32)) (31) contains $V(\phi)$, whereas (38) contains only $V^{\prime}(\phi)$. This is important, for instance, for Higgs fields, where $V(\phi)$ develops a vacuum expectation value, a constant $\langle V(\phi)\rangle$. In (31) this introduces a cosmological constant, a term $g^{\mu \nu}\langle V(\phi)\rangle$, in the last equation. From the constants involved, the universe should shrink to the size of an orange $\left.{ }^{3}\right)$. This shrinking could be countered by adding to the Lagrangian in (30) a term $\sqrt{g} \Lambda$, with $2 \Lambda=-\langle V(\phi)\rangle$, i.e. by adding an opposite cosmological constant from the beginning. Such a procedure seems, however, artificial. For eq. (38), which contains only $V^{\prime}(\phi)$ such a shrinking does not occur. This is true even if one replaces $S^{\mu \nu}$ in (38) by $T^{\mu \nu}$ of (32), which is allowed as $S^{\mu \nu}$ and $T^{\mu \nu}$ differ by a term proportional to $g^{\mu \nu}$ which is filtered out in (38).

Because of the Lagrange multiplier in that action, one cannot add a term into (39) which represents a cosmological constant. However, in solving 
equations (38) an integration constant $\Lambda$ appears which plays the role of a cosmological constant in the equations*.

Using the identity

$$
D_{\mu}\left(R^{\mu \nu}-\frac{1}{2} g^{\mu \nu} R\right) \equiv 0 .
$$

and with

$$
D_{\mu} S^{\mu \nu}=\frac{1}{2} D^{\nu}\left(V(\phi)+\frac{1}{2} D^{\alpha} \phi D_{\alpha} \phi\right),
$$

from (37), (38), one finds

$$
D^{\nu}(R-S-2 V(\phi))=0 \text {. }
$$

With the general solution

$$
R-S-2 V(\phi)=\Lambda,
$$

where $\Lambda$ is an arbitrary constant. Substituting into (38) the third equation may now be written as

$$
R^{\mu \nu}-\frac{1}{2} g^{\mu \nu} R-T^{\mu \nu}-\Lambda g^{\mu \nu}=0,
$$

where $T^{\mu \nu}$ is determined by (32), up to an arbitrary term $g^{\mu \nu} c$, which may be absorbed in the free constant of integration $\Lambda$ in (43). This integration constant is, in principle, not related to the vacuum expectation value of $T^{\mu \nu}$. Our entire derivation was based on the validity of the Poincare group, which means that space time must be asymptotically flat (but not necessarily locally flat!). Therefore the only choice for $\Lambda$ consistent with that derivation is that one which cancels whatever constant $\times g^{\mu \nu}$ is contained in $T^{\mu \nu}$.

The equations (38) of which, as we have seen, the third one may be replaced with (43), but with an arbitrary free constant $\Lambda$, are just Einstein's equations with an arbitrary cosmological constant $\Lambda$ and with the additional condition $\operatorname{det}\left(g_{\mu \nu}\right)=-1$. For any solution to Einstein's equations one may always locally choose coordinates for which this latter condition is satisfied. Several familiar solutions allow coordinates which globally satisfy $\operatorname{det}\left(g_{\mu \nu}\right)=-1$. An example is the Schwarzschild solution, written in Cartesian coordinates it has $g \equiv$ $-\operatorname{det}\left(g_{\mu \nu}\right)=1$ and thus it is a solution to (38) with $T^{\mu \nu}=0=\Lambda$. Similarly, the Friedmann solution allows a set of coordinates for which $g=1$. This appears to be a general property of solutions to Einstein's equations. To be specific: under very general conditions any solution can be covered with coordinate patches in each of which the constraint $g=1$ is satisfied, this may be shown as follows $\mathbf{s}^{19}$.

\footnotetext{
* That a parameter which is not present in the original Lagrangian finds its way into a theory is not new. Such theories include the massive Schwinger model, and quantum chromodynamics in four dimensions (When instanton effects are taken into account). See e.g., S. Coleman's 1977 Erice lectures, ed. by A. Zichichi (Plenum Press, N.Y., 1979).
} 
Suppose that the $\left\{x^{\mu}\right\}$ are some given set of coordinates for which $\sqrt{g^{\prime}} \neq 1$, and one wants to find coordinates $\left\{x^{\mu}\right\}$ for which $\sqrt{g}=1$. We can, for example, take $t=t\left(t^{\prime}, x^{\prime i}\right)$ and $x^{i}=x^{i}(i=1,2,3)$ such that

$$
t=\int^{t^{\prime}} \sqrt{g^{\prime}\left(t^{\prime \prime}, x^{\prime \prime}\right)} d t^{\prime \prime} \text {. }
$$

$\sqrt{\bar{g}}$ is a scalar density, so it transforms as

$$
\sqrt{g^{\prime}}=\operatorname{det}\left(\frac{\partial x^{\mu}}{\partial x^{\prime \nu}}\right) \sqrt{g}
$$

For the change of coordinates from $x^{\prime}$ to $x$, given by (44a), one has

$$
\operatorname{det}\left(\frac{\partial x^{\mu}}{\partial x^{\prime \nu}}\right)=\frac{\partial t}{\partial t^{\prime}}=\sqrt{g^{\prime}}
$$

Substituting this in (44b) gives $\sqrt{g}=1$.

\section{Discussion, three further aspects of our point of view}

To summarize, the starting point of this work is based on the validity of the Poincare group and is deeply rooted in the particle concept of the graviton. Invariance of quantum mechanics for the Poincare group brings to fore the importance of the little group. While the little group consideration yields the conventional gauge concept for a massless spin-1 particle, it gives rise to a more restricted form of gauge transformations for the massless spin-2 case. In short, one is led to a theory with a constraint. But one can show ${ }^{10}$ ) that, under general conditions, the constraint can be satisfied.

For pure gravity, our theory appears to be equivalent to the standard theory. ${ }^{11}$ ). But even for this case the little group consideration has been valuable in that it sheds light on a different view of the consmological constant (be it empirically found to be zero or not). Within the new framework, that constant appears as an integration constant (hence a free parameter) rather than in the Lagrangian. The difference between the usual formulation of the theory of gravity and the present one may perhaps be illustrated by a diagram. In this diagram one represents solutions to Einstein's equations, the cosmological constant $\Lambda$ is plotted horizontally and $g$ vertically (this is an oversimplification as $-\operatorname{det} g_{\mu \nu}$ depends on $x$ ). The usual action allows solutions with only one value of $\Lambda$, but with any value of $g$, i.e. it corresponds to a vertical line in the diagram. The action (38), however, corresponds to a horizontal line, having solutions for all $\Lambda$, but each solution in a gauge $g=1$, i.e. in a coordinate system with equal unit cells. 
Next, let us discuss three further aspects of the present framework.

(a) It is instructive to view the above approach of the gravitational theory from another perspective. To do that let us start with the first order action of Einstein's theory:

$$
S=\int(\mathrm{d} x) \sqrt{g}\left(\Gamma_{\mu \sigma}^{\alpha} \Gamma_{\nu \alpha}^{\sigma}-\Gamma_{\mu \nu}^{\sigma} \Gamma_{\alpha \sigma}^{\alpha}\right) g^{\mu \nu},
$$

and substitute

$$
g_{\mu \nu}=\Omega^{2} \tilde{g}_{\mu \nu},
$$

then the action (45) becomes $\left.{ }^{12}\right)\left(\operatorname{det}\left(\tilde{g}_{\mu v}\right) \equiv-\tilde{g}\right)$

$$
S=\int(\mathrm{d} x) \sqrt{\overline{\mathrm{g}}}\left[\Omega^{2} \tilde{R}+6 \partial_{\mu} \Omega \tilde{\mathrm{g}}^{\mu \nu} \partial_{\nu} \Omega\right],
$$

where $\tilde{R}$ is the scalar curvature defined via $\tilde{g}_{\mu \nu} \Omega$ is called the conformal factor ${ }^{12}$ ). One must not regard $\Omega$ as a physical scalar field since its derivative term has the wrong sign. Due to that term, one can see that the Euclidean version of the action (47a) may be as negative, as one wants, by choosing a rapidly varying $\Omega$; and in this connection there appear some difficulties in carrying out the path integrals ${ }^{13}$ ). In (46), one can choose det $\tilde{g}_{\mu \nu}=-1$ without loss of generality so that the action (47a) becomes

$$
S=\int(\mathrm{d} x)\left[\Omega^{2} \tilde{R}+6 \partial_{\mu} \Omega \tilde{g}^{\mu \nu} \partial_{\nu} \Omega+L(\tilde{g}-1)\right] .
$$

Now one realizes that the formulation presented in this paper is obtained by setting $\Omega=1$ ! The reference to the conformal factor is gone, so is the (apparent) difficulty it causes. (Indeed we could turn the argument around and use this reasoning to motivate the new formulation.)

(b) Another intriguing (and potentially useful) result of the little group viewpoint is that the theory of gravity with the constraint can in fact be described by a Lagrangian that is a polynomial of $g^{\mu \nu}$ of order $9 .^{*}$ We have**, using the action (39), which has equations of motion (38),

$$
\begin{aligned}
\mathscr{L}_{\text {gravity }} & =R+L(g-1) \\
& =-\partial_{\mu} \partial_{\nu} g^{\mu \nu}-\frac{1}{4} g_{\mu \nu} g_{\alpha \beta} g^{\tau \sigma} \partial_{\tau} g^{\mu \alpha} \partial_{\sigma} g^{\nu \beta}+\frac{1}{2} g_{\mu \nu} \partial_{\alpha} g^{\mu \sigma} \partial_{\sigma} g^{\nu \alpha}+L(g-1),
\end{aligned}
$$

where

$$
g_{\mu \nu}=\frac{1}{3 !} \epsilon_{\mu \alpha \beta \gamma} \epsilon_{\nu \sigma r \eta} g^{\beta \tau} g^{\alpha \sigma} g^{\nu \eta},
$$

* J.W. York (private communication) has already noted that $\tilde{R}$ of (47b) can be written in the form of a polynomial of finite order.

** If we use $g_{\mu \nu}$ as the basic variable, then the Lagrangian becomes a polynomial of order 11. 


$$
g=-\operatorname{det} g^{\mu \nu}=\frac{-1}{4 !} \epsilon_{\mu \alpha \beta \gamma} \epsilon_{\nu \sigma \beta \gamma} g^{\mu \nu} g^{\alpha \sigma} g^{\beta \tau} g^{\gamma \eta}
$$

The first term $-\partial_{\mu} \partial_{\nu} g^{\mu \nu}$ in the Lagrangian (48) can be omitted if $\partial_{\mu} g^{\mu \nu}=0$ on the boundary. Formulated in this way the theory has a remarkable resemblence to the nonlinear $\sigma$-model $\left.{ }^{14}\right)^{*}$.

(c) Finally, we observe that the apparent disagreement for the measure on the space of all metrics between some workers of relativity ${ }^{15}$ ), viz.

$$
D[g]=\prod_{x} \prod_{a \geqslant b} \mathrm{~d} g_{a b},
$$

versus

$$
D[g]=\prod_{x} \prod_{a \geqslant b} \mathrm{~d} g_{a b}(g)^{-5 / 2},
$$

does not appear to be of a serious nature since $(g)^{-5 / 2}$ now equals unity.

To be complete, let us note that there is a connection to conformal super-gravity ${ }^{16}$ ). Note also that Hawking ${ }^{17}$ ) has used a Lagrange multiplier similar to the one considered in this paper. However, it seems that this was done for an essentially different purpose. Finally there is an interesting paper by Lanczos ${ }^{18}$ ).

\section{Acknowledgements}

We thank P. van Nieuwenhuizen, L.C. Biedenharn, T. Kephart, S. Raby and Th.W. Ruygrok for discussions, and particular R. van Damme and $M$. Green for help in computing the Green's functions. Special thanks are due also to Paul Federbush and L.H. Ford for a crucial remark. ${ }^{19}$ ). We thank J.W. York for many very useful conversations and for the original stimulus for this work. This work was supported by F.O.M., D.O.E. and the Alfred Sloan Foundation.

\section{References}

1) E.P. Wigner, Ann. Math. 40 (1939) 149

2) See, for instance, M. Veltman, in Methods in Field Theory, R. Balian and J. Zinn-Justin, eds. (North-Holland, Amsterdam, 1976) p. 266.

3) A.D. Linde, Pisma Zh. Eksp. Teor. Fiz. 19 (1974) 320 [JETP Lett. 19 (1974) 183]. M. Veltman Phys. Rev. Lett. 34 (1975) 777.

4) Also see S. Weinberg, Phys. Rev. B 135 (1964) 1049.

* To (48) one can easily add higher order terms in the Riemann curvature tensor. 
5) R.P. Feynman, Lectures on Gravitation (Caltech, 1962); D.G. Boulware and S. Deser, Ann. Phys. N.Y. 89 (1975) 193.

6) H. van Dam and M. Veltman, Nucl. Phys. B22, (1970) 397.

7) J. Schwinger, Phys. Rev. 173 (1968) 1264.

8) J. Schwinger, Am. J. Phys. 42 (1973) 510.

9) Compare ref. 2, p. 272, or G. 't Hooft and M. Veltman, Ann. Inst. H. Poincaré 20 (1974) 69.

10) The proof given here is first due to Paul Federbush (private communication), and independently due to L.H. Ford (private communication.)

11) See, however, a forthcoming paper by J.W. York.

12) H. Weyl, Raum Zeit Materie (Springer, Berlin, 1923) p. 131.

See also J. Schwinger, Particles, Sources and Fields, vol. 1 (Addison-Wesley, Reading, Mass., 1970) p. 392.

13) G. W. Gibbons, S. W. Hawking and J. Perry, Nucl. Phys. B138 (1978) 141.

14) M. Gell-Mann and M. Levy, Nuovo Cimento 16 (1960), 705.

15) Eq. (51) is proposed by, e.g., C.W. Misner, Rev. Mod. Phys. 29 (1957) 497, and L.D. Fadeev and V.N. Popov. Sov. Phys. Usp. 16 (1974) 777. Eq. (50) is mentioned by S.W. Hawking, talk given in GR8, Waterloo, Ontario, Aug. 1977; also see his Caltech OAP-506 prepring, Oct. 1977. But the published version of that preprint, Phys. Rev. D18 (1978) 1747, does not contain that result. We bring up the discussion here because there is still confusion on this issue in the relativity community.

16) P. van Niewenhuizen, Phys. Rep. 68 (1981), in particular p. 333.

17) S.W. Hawking, in Recent Developments in Gravitation (Cargese 1978), M. Levy and S. Deser, eds. (Plenum, New York, 1979) p. 167.

18) C. Lanczos, Rev. Mod. Phys. 34 (1962) 379. 